\title{
CSCW-TRABALHO COOPERATIVO SUPORTADO POR COMPUTADOR
}

\section{Eulina X. Botelho ${ }^{1}$ e Jorgiano M. B. Vidal $^{2}$}

${ }^{1}$ Gerência de Tecnologia da Informação - CEFET-RN. eulinabotelho@digizap.com.br

${ }^{2}$ Professor da Gerência de Tecnologia da Informação - CEFET-RN. jorgiano@cefetrn.br

Recebido em outubro de 2004 e Aceito em abril de 2005

\section{RESUMO}

Neste artigo iremos tratar da área da computação que se preocupa com a evolução rápida da informação e dos potenciais novos para uma comunicação entre povos. Os aspectos chaves são a disponibilidade aumentada de redes de computador e a tendência para o trabalho cooperativo. Uma ênfase principal para sistemas aplicados na sustentação do computador para o trabalho da equipe. As atividades nesse domínio são sabidas pelas noções do groupware ou do trabalho cooperativo suportado por computador (CSCW).

Palavras-chave: colaboração, cooperação, cscw, groupware. 


\section{CSCW-TRABALHO COOPERATIVO SUPORTADO POR COMPUTADOR}

\section{INTRODUÇÃO}

Os precários meios de comunicação do passado não permitiam que pessoas interagissem a não ser por meio da presença física dos envolvidos em um mesmo lugar. As pessoas precisavam se locomover para se comunicar e interagir umas com as outras. Entretanto, no mundo de hoje, a complexidade e o tamanho das tarefas do mundo atual exigem e exigirá maior interação entre as pessoas. Cada vez mais a solução de problemas complexos passa pela multi-disciplinaridade; pessoas com diferentes formações precisam cooperar para atingir os resultados esperados. Essas necessidades permitem prever um desenvolvimento crescente dos meios de comunicação.(M. C. R. Cavalcanti et al.,1995)

A informação passou a ser um importante e decisivo fator de produção. Hoje, ser competitivo requer, consistentemente, maior velocidade na tomada de decisões de gerência, no desenvolvimento de produtos e no atendimento ao cliente (segundo B. S. Backer e D. Carter,1991). Esse quadro, juntamente com fatores como a disseminação das redes de computadores e sistemas distribuídos (segundo A. S. Souza, L. G. Golendziner, 1996), tem incentivado cada vez mais a formação de grupos de trabalho. Estes grupos agem no sentido de solucionar problemas cada vez mais complexos e desafiadores, com maior qualidade e velocidade, através da soma dos talentos do grupo, com cada membro contribuindo com sua parte para o produto final (segundo J. V. Lima e M. K. Pinheiro, 1999). É justamente nesse ambiente que a área de Trabalho Cooperativo (ou CSCW - Computer Supported Cooperative Work) tem se destacado, uma vez que seus objetivos são justamente os de estudar o trabalho em grupo e meios de apoiá-lo sob vários aspectos, com a intenção de aumentar a produtividade do grupo (K. Spurr et. al., 1994).

\section{CONCEITOS IMPORTANTES}

\section{Trabalho Colaborativo}

Trabalho Colaborativo é o que envolve duas ou mais pessoas de forma colaborativa, compartilhando informações. Para haver um trabalho colaborativo é necessário que os indivíduos possuam habilidades para trabalho colaborativo em grupo, base de dados organizada e padronizada eficiência de comunicação, espírito de coletividade e bom relacionamento (segundo Marcelo e Morandini, 2000). Normalmente, a comunicação entre grupos de pessoas traz mais benefícios do que o incentivo à produtividade individual.

Ultimamente, tem-se dado grande ênfase à qualidade e à satisfação das necessidades dos consumidores, abrindo-se cada vez mais espaços para os trabalhos colaborativos com envolvimento de equipes multifuncionais, com grande destaque aos sistemas de comunicação.

\section{Sistema Cooperativo}

“Espaço para compartilhamento de idéias e aprendizado de experiências...” (Scherlis, 1996).

De trabalho cooperativo tem como resultado produtos de informação como decisões, concepção, análise, minimização de perda de informação e opera com elevados níveis de detalhe (Scherlis, 1996). 
A aprendizagem cooperativa pode revelar-se importante para uma educação completa, justificando a introdução das designadas Tecnologias de Informação e Comunicação (ICT) para o seu suporte.

\section{CSCW}

Computer Supported Cooperative Work vem ao encontro à necessidade atual de interação entre pessoas que estão geograficamente distantes, fornecendo um suporte computacional para que as pessoas possam interagir cooperativamente [M. C. R. Cavalcanti et al.,1995].

A área de CSCW aborda não apenas o projeto de sistemas que apóiam o trabalho de usuários, mas também os efeitos que a tecnologia acarreta no comportamento de trabalho desses usuários. Para suportar o trabalho cooperativo foi criada uma tecnologia para gerar sistemas, chamada Groupware, o que pode ser definido como (G. L. Rein et. al., 1991): "sistema baseado em computador que suporta grupos de pessoas engajadas em uma tarefa comum (ou ideal) e que fornece uma interface para um ambiente compartilhado”.

\section{Requisitos Do CSCW}

Os requisitos das ações e atividades em um CSCW referem-se às funcionalidades do sistema. Tais funcionalidades podem estar divididas em gerais e específicas. Estes requisitos podem ser considerados básicos não apenas para ambientes de groupware (CSCW), mas para quaisquer interações humano-computador. Tais requisitos de acordo (Schneiderman, B., 1992), serão descritos abaixo.

Os requisitos gerais são:

- Identidade individual dos membros: saber quem está logado;

- Posição do usuário: nomes e / ou ícones visíveis na tela;

- Conhecimento: outros integrantes saibam da presença de um usuário no grupo;

- Visão pessoal ou padrão: visão especificada por cada usuário para um problema;

- Visão convergente: princípio WYSIWIS (What You See Is What I See - O que você vê é o que eu vejo) é a mais recomendada;

- Visões particular e pública: partes podem ser vistas por grupos de usuários específicos;

- Controle da palavra: algumas situações necessitam de somente um usuário manipulando um aplicativo ou emitindo opiniões;

- Controle de acesso: direitos de acesso para cada usuário;

- Atualização e sincronismo: para não haver divergências na edição e / ou entrada e saída de dados;

- Coordenação da informação: coordenação de atividade ou grupo de atividades, aumentando a eficiência.

Já os requisitos específicos estão diretamente ligados às aplicações em si e são basicamente os mesmos requisitos de interface de qualquer aplicação e dispositivo. 


\section{GROUPWARE}

Software que apóia o Trabalho em Grupo, o groupware costuma ser usado quase como sinônimo de CSCW, porém possuem diferenças, pois enquanto CSCW é usado para designar a pesquisa na área do trabalho em grupo e como os computadores podem apoiá-lo, o groupware tem sido usado para designar a tecnologia (hardware e /ou software) gerada pela pesquisa em CSCW. (Náutilus Cirney Carneiro et. al., 2000)

Um dos requisitos fundamentais de sistemas de groupware é que os sistemas sejam altamente configuráveis, para se adaptarem às necessidades dos usuários.

Groupware também pode ser definido como a aglutinação de diferentes tecnologias em sistemas computacionais que objetivam apoiar a cooperação entre indivíduos que são parte de uma equipe. Torna o trabalho em grupo mais eficiente e possibilita certos tipos de tarefas em grupo que seriam impossíveis (ou quase) de ser realizada sem o suporte computacional.( Isabelle Betero, 1999)

O groupware utiliza ferramentas que ajudam as pessoas a trabalharem juntas mais fáceis e eficazmente, tais como:

Comunicação -Ajudar pessoas a compartilhar informações.

Coordenação - Ajudar pessoas a coordenar seus papéis pessoais com os outros.

Colaboração - Ajudar pessoas a trabalhar juntas.

\section{EXTREME PROGRAMMING - XP}

Extreme Programming (XP) é um processo de desenvolvimento que possibilita a criação de software de alta qualidade, de maneira ágil, econômica e flexível.( Vinícius Manhães Teles, 2004)

O XP concentra os esforços da equipe de desenvolvimento em atividades que geram resultados rapidamente na forma de software intensamente testado e alinhado às necessidades de seus usuários. Além disso, simplifica e organiza o trabalho combinando técnicas comprovadamente eficazes e eliminando atividades redundantes. Por fim, reduz o risco dos projetos desenvolvendo software de forma iterativa e reavaliando permanentemente as prioridades dos usuários.

Práticas do XP.( Klaus Wuestefeld, 2001)

- The Customer is Always Available - O cliente está sempre disponível para resolver dúvidas, alterar o escopo de uma iteração e definir prioridades.

- Metaphor - O time se comunica sobre o software em termos de uma metáfora, caso consiga encontrar uma boa.

- Planning Game - Os jogadores do jogo do planejamento são o cliente e os técnicos. O objetivo: colocar em produção as funcionalidades de maior valor possível durante o decorrer do jogo.

- Small Releases - O software é entregue em pequenas versões para que o cliente possa obter o seu ganho o mais cedo possível e para minimizar riscos. 
- Acceptance Tests - São definidos pelo usuário e são os critérios de aceitação do software.

- Test First Design - Primeiro são escritos os testes, depois é feita a implementação e por último trabalha-se o design.

- Continuous Integration - Os diversos módulos do software são integrados diversas vezes por dia e todos os testes unitários são executados. O código não passa até obter sucesso em 100\% dos testes unitários.

- Simple Design- O código está, a qualquer momento, na forma mais simples que passe todos os testes.

- Refactoring - A cada nova funcionalidade adicionada, é trabalhado o design do código até ficar na sua forma mais simples.

- Pair Programming - Todo código de produção é desenvolvido por duas pessoas trabalhando com o mesmo teclado, o mesmo mouse e o mesmo monitor.

- Move People Around - As duplas de programação são revezadas em média a cada 2h.

- Collective Code Ownership - E equipe como um todo é responsável por cada arquivo de código. Não é preciso pedir autorização para alterar qualquer arquivo.

- Coding Standards - Todo código é desenvolvido seguindo um padrão.

- 40 Hour Week - Trabalhar por longos períodos é contraproducente.

Valores: (Klaus Wuestefeld, 2001)

- Simplicidade: Faça o mais simples que possa funcionar, e torne as mudanças fáceis e de baixo custo.

- Comunicação: Forçada por práticas que obrigam a interação (jogo de planejamento, vocabulário comum, duplas).

- Feedback: Todo problema é evidenciado o mais cedo possível para que possa ser corrigido o mais cedo possível. Toda oportunidade é descoberta o mais cedo possível para que possa ser aproveitada o mais cedo.

- Coragem: Base sólida (cobertura de testes, manutenção preventiva, integração contínua, código simples) proporciona segurança e agressividade.

\section{ARQUITETURA}

O Trabalho Cooperativo Suportado por Computador (Computer Supported CooperativeWork CSCW) surgiu como uma alternativa de exploração das virtudes do trabalho em grupo, e está se tornando uma forte tendência tanto no área acadêmica quanto na área comercial, gerando uma mudança no comportamento dos indivíduos (Bentley, R., 1992). Sob o ponto de vista educacional, CSCW resgata questões metodológicas importantes no que concerne à modelagem e implementação de ambientes de ensino-aprendizagem computadorizados; sob o ponto de vista comercial CSCW oferece um novo paradigma de aplicações que fornece subsídio para uma tecnologia baseada na cooperação. Dentro desse contexto, o presente trabalho apresenta a concepção e implementação de uma arquitetura distribuída para o suporte à gerência de objetos distribuídos para ser utilizado em ambientes cooperativos (groupware) na Internet.

O principal objetivo na concepção desta arquitetura é fornecer uma camada de software para suporte a aplicações colaborativas (groupware), de forma que estas sejam facilmente construídas, sem que as mesmas precisem se preocupar com a distribuição e armazenamento de objetos criados e utilizados pelos usuários destas aplicações. 


\section{Arquitetura Proposta}

Nesta seção será apresentado uma Arquitetura Genérica para Aplicações Groupware (GAGA Generic architecture for Groupware Applications). O sistema GAGA foi desenvolvido de forma a prover as seguintes funcionalidades (Tanebaum, A. S., 1996):

- Fornecer mecanismos para a transferência de informações (objetos) entre o local de armazenamento para a aplicação groupware solicitante e vice-versa;

- Possibilitar a procura e recuperação de informações (objetos) que estão armazenados de forma distribuída;

- Permitir a utilização das informações (objetos) recuperadas pela aplicação groupware;

- Permitir o compartilhamento das informações (objetos) que estão armazenada de forma distribuída, por diversos usuários;

- Manter a consistência das informações (objetos) armazenadas na base de dados distribuída.

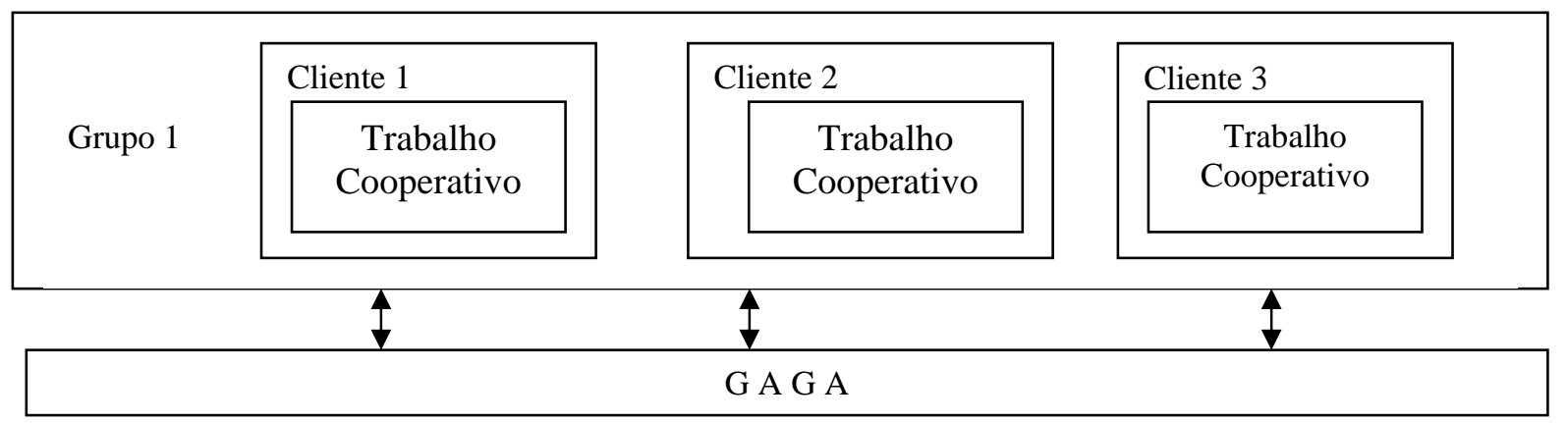

A Figura 1 exemplifica a arquitetura do GAGA para contemplar as funcionalidades supracitadas.

Cada aplicação groupware (cliente) é um programa que executa suas funções (funcionalidades) pertinentes ao objetivo a que se propõe. Caso essa aplicação necessite de um suporte ao trabalho cooperativo, o sistema GAGA supre essa necessidade com uma camada de software que fornece características para que o trabalho cooperativo possa se realizar. Outra funcionalidade do sistema GAGA é o de o armazenamento físico e recuperação das informações (objetos) que um cliente necessita compartilhar com outros os clientes. Estes objetos são utilizados pelos clientes de forma que a cooperação entre os mesmos seja possível.

O GAGA se encarrega de fornecer a comunicação entre os clientes, como meio de cooperação, caso a cooperação necessite ser síncrona, toda vez que um objeto é modificado, todos os clientes são notificados. O GAGA possui também mecanismos para fornecer cooperação assíncrona através do armazenamento de informações (objetos), quando um objeto é modificado por um cliente, o sistema GAGA não informa os demais clientes, mas atualiza o objeto no espaço de trabalho para que os clientes possam requisitar informações sobre objetos modificados quando necessitarem.

\section{Arquitetura do Sistema GAGA}

O sistema GAGA consiste dos seguintes componentes:

- WSServer: servidor do espaço de trabalho compartilhado, responsável por fornecer os mecanismos para a realização do trabalho cooperativo;

- ObjectServer: servidor de objetos, responsável quando necessário, pelo armazenamento dos objetos em disco; 
- WSManagerServer: gerente dos servidores de objetos e dos servidores dos espaços compartilhados,responsável em gerenciar vários WSServer e ObjectServer;

- $\quad$ as aplicações.

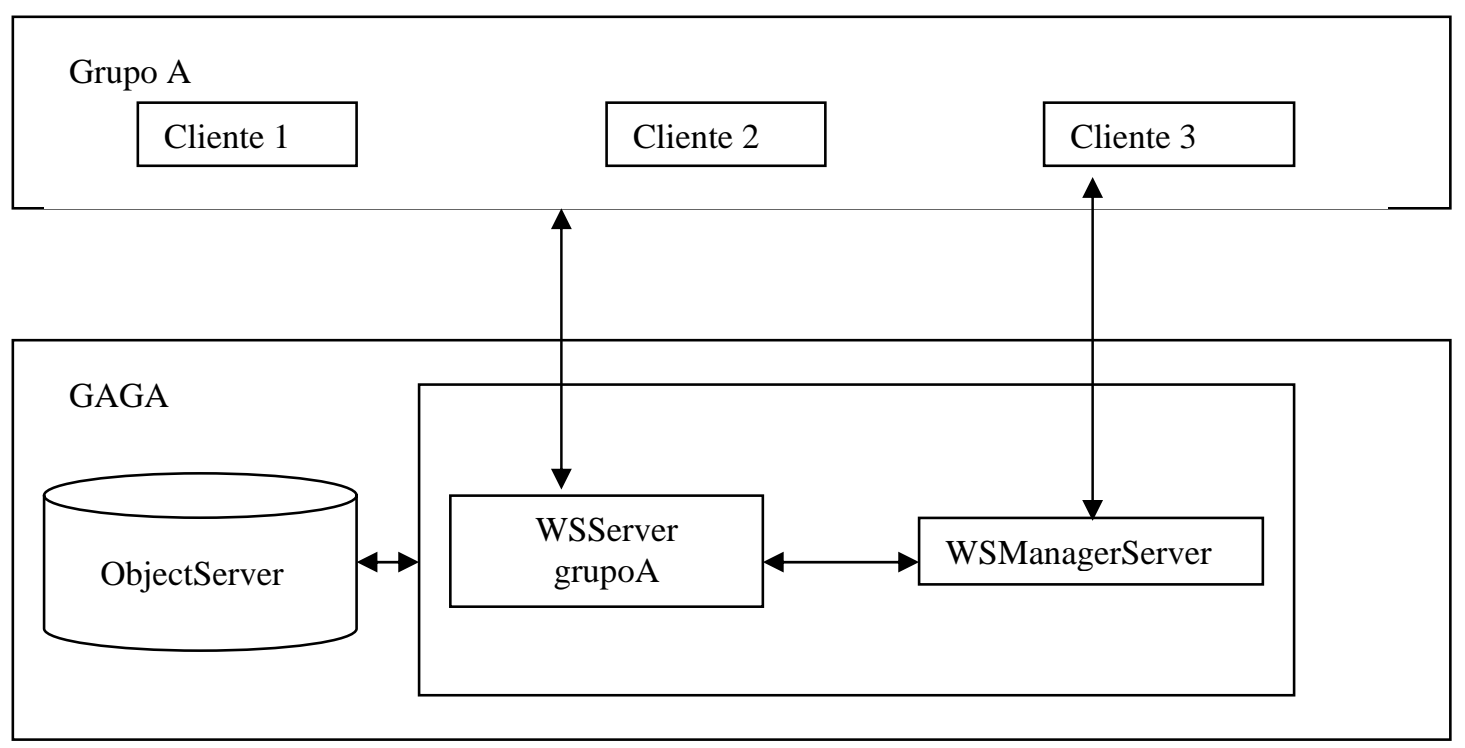

Figura 2: Arquitetura do GAGA.

Cabe salientar que cada cliente pode participar de várias cooperações ao mesmo tempo, ou seja, pode ter acesso a diversos espaços compartilhados ao mesmo tempo. Podem-se também ter vários WSServer por WSManagerServer. Cada WSServer é identificado por um nome único pelo WSManagerServer.

Outra característica é a possibilidade da utilização de vários ObjectServer simultaneamente para cada WSManagerServer, mas também com nomes diferentes. A utilização de diversos servidores de objetos tem como objetivos principais, a melhora na performance, e também a possibilidade de tolerar falhas caso um dos servidores não esteja funcionando em um determinado momento. O mesmo pode ser dito a respeito dos servidores de espaços compartilhados.

Quanto ao gerente, no momento o mesmo é um ponto centralizador, o que não afeta a performance, pois o mesmo realiza basicamente atividades de busca de servidores.

O objetivo desta arquitetura é criar um mecanismo para ser utilizado por aplicações groupware facilitando a cooperação entre os usuários. Desse modo, a arquitetura não provê cooperação diretamente, e sim, fornece meios para que as aplicações possam cooperar.

\section{CONCLUSÃO}

Uma crescente parte do trabalho das empresas e instituições não é mais realizada individualmente, com uma pessoa trabalhando sozinha até completar as tarefas. O trabalho é cada vez mais realizado colaborativamente. Como também, o crescente número de sistemas 
complexos que surgem exigem a necessidade de interação entre as pessoas que nele trabalham. Esta tendência se deve parcialmente ao aumento de complexidade das tarefas, que passam a requerer habilidades multidisciplinares, e aos novos paradigmas de trabalho, que envolvem diversos setores da empresa, ou até mesmo outras empresas, trabalhando conjuntamente nas diversas fases de elaboração de um produto ou desenvolvimento de um projeto. Portanto, surge o CSCW (Computer Supported Cooperative Work), como área da computação que se preocupa coma interação entre as pessoas na relação espaço e tempo e com a complexidade dos novos sistemas.

\section{REFERÊNCIAS BIBLIOGRÁFICAS}

A. S. Souza, L. G. Golendziner. Um Estudo Sobre Trabalho Cooperativo Suportado Por Computador (CSCW). Trabalho individual. Porto Alegre, CPGCC/UFRGS, 1996.

B. S. Backer, D. Carter. Concurrent Engineering: The Product Development Environment for the 1990s. Vol.1. Mentor Graphics Corporation, 1991.

Bentley, R. An architecture for tailoring cooperative multi-user displays. Em Proceedings of 4th Conference on Computer-Supported Cooperative Work, Out./Nov. 1992.

C. A. Ellis; G. L. Rein e S. J. Gibbis e. Groupware: Some issues and experiences. Communications of the ACM (34) 1, Jan 1991, pp. 38-58.

Isabelle Betero, “A importância cultural na implantação de tecnologias Groupware”. Ponta Grossa, 1999.

J. V. Lima e M. K. Pinheiro. Edição Cooperativa de Hiperdocumentos na WWW. Trabalho individual. Porto Alegre, CPGCC/UFRGS, 1999.

K. Spurr, P. Layzell, L. Jennison, N. Richards. Computer Support for Co-Operative Work. Chichester, John Wiley, 1994.

Klaus Wuestefeld. Xispe: Extreme Programming, 2001.

M. C. R. Cavalcanti, M. L. M. Campos e M. R. S. Borges. Suporte por Computador ao Trabalho Cooperativo. In XIV Jornada de Atualização em Informática, Canela. Julho/Agosto 1995.

Marcelo e Morandini. Critérios e Requisitos para Avaliação da Usabilidade de Interfaces em Groupware - CSCW. Faculdade de Engenharia Elétrica e de Computação-FEEC Unicamp.

Náutilus Cirney Carneiro, Paulo Blauth Menezes, Rodrigo Quites Reis. Especificação Formal de uma Ferramenta de

Schneiderman, B. Designing the User Interface: Strategies for Effective Human-Computer Interaction; Addison-Weslwy, 1992.

Tanebaum, A. S. Distributed Operating Systems. Prentice-Hall, 1996.

Trabalho Colaborativo através da Composição de Objetos, 2000.

Vinícius Manhães Teles, “Extreme Programming”, 13/01/2004. 